\title{
The Epigenetic Clock at Birth: Associations With Maternal Antenatal Depression and Child Psychiatric Problems
}

\author{
Anna Suarez, MA, Jari Lahti, PhD, Darina Czamara, PhD, Marius Lahti-Pulkkinen, PhD, \\ Anna K. Knight, BS, Polina Girchenko, MS, Esa Hämäläinen, MD, Eero Kajantie, MD, \\ Jari Lipsanen, MA, Hannele Laivuori, MD, Pia M. Villa, MD, Rebecca M. Reynolds, MD, \\ Alicia K. Smith, MD, Elisabeth B. Binder, MD, Katri Räikkönen, PhD
}

Objective: Maternal antenatal depression may compromise the fetal developmental milieu and contribute to individual differences in aging and
disease trajectories in later life. We evaluated the association between maternal antenatal depression and a novel biomarker of aging at birth, namely
epigenetic gestational age (GA) based on fetal cord blood methylation data. We also examined whether this biomarker prospectively predicts and
mediates maternal effects on early childhood psychiatric problems.

Method: A total of 694 mothers from the Prediction and Prevention of Preeclampsia and Intrauterine Growth Restriction (PREDO) Study provided information on history of depression diagnosed before pregnancy; 581 completed the Center for Epidemiological Studies Depression Scale throughout pregnancy, and 407 completed the Child Behavior Checklist at child's age 3.7 years (SD =0.75 year). DNA methylation (DNAm) GA of fetal cord blood DNA was based on the methylation profile of 148 selected cytosine linked to guanine by phosphate (CpG) sites. Epigenetic GA was calculated as the arithmetic difference between DNAm GA and chronological GA and adjusted for chronological GA.

Results: Maternal history of depression diagnosed before pregnancy (mean difference $=-0.25 \mathrm{SD}$ units, $95 \% \mathrm{CI}=-0.46$ to -0.03 ) and greater antenatal depressive symptoms ( -0.08 SD unit per 1-SD unit increase, 95\% CI $=-0.16$ to -0.004$)$ were associated with child's lower epigenetic GA. Child's lower epigenetic GA, in turn, prospectively predicted total and internalizing problems and partially mediated the effects of maternal antenatal depression on internalizing problems in boys.

Conclusion: Maternal antenatal depression is associated with lower epigenetic GA in offspring. This lower epigenetic GA seems to be associated with a developmental disadvantage for boys, who, in early childhood, show greater psychiatric problems.

Key words: epigenetic clock, gestational age, cord blood, antenatal depression, childhood psychiatric problem

J Am Acad Child Adolesc Psychiatry 2018;57(5):321-328.

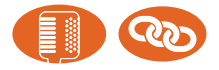

M aternal depression complicates up to $20 \%$ of pregnancies. ${ }^{1}$ Depression not only hinders the health and well-being of the pregnant mother, but is also associated with adverse perinatal and developmental outcomes in her child. The adverse outcomes include prematurity ${ }^{2}$ and increased risks of poorer cognition and psychiatric problems later in life. ${ }^{1,3-5}$ Findings from these studies are compatible with the Developmental Origins of Health and Disease (DOHaD) framework, which suggests that in utero exposure to environmental adversities may alter the structure and function of developing cells, tissues, organs, and physiological feedback systems, leading to individual differences in aging and disease trajectories that persist throughout the lifespan. ${ }^{3}$

Mounting evidence suggests that exposure to prenatal environmental adversity may be embedded in epigenetic alterations in the offspring, such as in DNA methylation (DNAm), which is characterized by the addition of a methyl group primarily to cytosine linked to guanine by phosphate (CpG) sites. DNAm can be used to generate aggregate markers of aging, such as the Hannum ${ }^{6}$ and the Horvath ${ }^{7}$ epigenetic age predictors. The Hannum age predictor is based on DNAm of $71 \mathrm{CpG}$ sites in whole blood of 19- to 101-year-old individuals, ${ }^{6}$ and the Horvath age predictor on
DNAm of 353 CpG sites of multiple tissues of 0 - to 100-year-old individuals. ${ }^{7}$ Both predictors are highly correlated with an individual's chronological age $(r>0.91)$. Having a higher DNAm age than chronological age, based on these molecular aging biomarkers, has been shown to predict disease trajectories and mortality more accurately than chronological age. ${ }^{8}$ However, it remains less clear whether exposure to prenatal environmental adversity is associated with epigenetic gestational age (GA) at birth or how epigenetic GA is associated with developmental problems later in life.

A recent study demonstrated that a higher epigenetic GA (higher DNAm GA than chronological GA), based on the Horvath and the Hannum epigenetic age predictors of cord blood methylation data, was associated with maternal smoking during pregnancy and delivery by cesarean section. ${ }^{9}$ These predictors are, however, not well suited for epigenetic age estimation at birth, because their correlation with chronological GA is nearly $0 .{ }^{9}$ A recent study generated a novel epigenetic GA biomarker based on fetal umbilical cord blood or newborn blood spots, ${ }^{10}$ which showed a high correlation with ultrasound-based GA. ${ }^{10}$ This study demonstrated that lower epigenetic GA (lower DNAm GA than 
chronological GA) at birth was associated with maternal socioeconomic disadvantage and low birth weight. We have extended these analyses by showing that lower epigenetic GA was associated with maternal insulintreated gestational diabetes mellitus in a previous pregnancy and in Sjögren syndrome, and higher epigenetic GA with a maternal age of more than 40 years at delivery, neonate's lower 1-minute Apgar score, and female sex. ${ }^{11}$

We are unaware of any previous studies that would have tested whether maternal antenatal depression and child psychiatric problems are associated with child's epigenetic GA at birth. The current study sought to determine whether maternal history of depression diagnosed before pregnancy and antenatal depressive symptoms were related to the child's epigenetic GA at birth based on fetal cord blood DNAm data. We also tested whether these conditions exerted additive effects on child's epigenetic GA, as not all individuals with a history of depression diagnosis report high antenatal depressive symptoms and vice versa, ${ }^{1}$ and whether the effects of antenatal depressive symptoms were gestation-stage specific. Furthermore, we tested whether child's epigenetic GA at birth predicted early childhood psychiatric problems, and whether child's epigenetic GA mediated the associations of maternal antenatal depression with child psychiatric problems. Finally, we tested whether the associations were moderated by child's sex, as sex biases exist in longevity and aging trajectories, ${ }^{12}$ epigenetic age based on the Horvath and the Hannum epigenetic age predictors, ${ }^{6,9}$ epigenetic GA at birth, ${ }^{11}$ and prevalence and etiology of psychiatric problems. ${ }^{1,3-5,13}$. The effects of maternal stress exposure during pregnancy ${ }^{3-5,14}$ and particularly antenatal depres$\operatorname{sion}^{4}$ on offspring developmental outcomes, have also shown sex specificity. Evidence in humans has shown that maternal antenatal depression was associated with a higher risk of depression in the offspring at 18 years of age, but only in girls. ${ }^{4}$ Yet, in one rodent study, maternal early pregnancy stress was associated with a depression-like behavioral phenotype in male but not in female offspring. ${ }^{14}$

\section{METHOD}

\section{Study Population}

The Prediction and Prevention of Preeclampsia and Intrauterine Growth Restriction (PREDO) Study comprises 1,083 pregnant women and their singleton children born in 2006 to 2010 in Finland. ${ }^{15}$ The recruitment took place in consecutive order when these women attended their first ultrasound screening at 12 to 13 weeks of gestation in hospital maternity clinics of the study. Of the recruited women, 973 had one or more risk factors for preeclampsia and intrauterine growth restriction (IUGR), and 110 had no known risk factors.

In total, we had 814 fetal umbilical cord blood samples with full information on genome-wide methylation and genotype passing through quality control (see Figure S1, available online). Of this entire sample, data on maternal depression diagnosed before pregnancy were available for 694 individuals, on maternal antenatal depressive symptoms for 581, and on childhood psychiatric problems at child's mean age of 3.7 years for 407. Table 1 describes the sample characteristics.

The Ethics Committees of the Helsinki and Uusimaa Hospital District and the participating hospitals approved the study protocol. Written informed consent was obtained from all participating women.

\section{Child DNA Methylation, Epigenetic, and Chronological GA at Birth}

Fetal cord blood samples were collected according to standard procedures. DNA was extracted at the National Institute for Health and
Welfare, Helsinki, Finland, and the Institute for Molecular Medicine Finland, University of Helsinki, Finland. Methylation analyses were performed at the Max Planck Institute of Psychiatry in Munich, Germany. DNA was bisulphite converted using the EZ-96 DNA Methylation kit (Zymo Research, Irvine, CA). Genome-wide methylation status of more than $485,000 \mathrm{CpG}$ sites was measured using the Infinium Human Methylation 450 BeadChip (Illumina Inc., San Diego, CA) according to the manufacturer's protocol. The arrays were scanned using the iScan System (Illumina Inc., San Diego, CA). The quality control pipeline was set up using the R-package minfi. ${ }^{16}$ Samples with maternal blood contamination were excluded $(n=9)$ according to a method described elsewhere. ${ }^{17}$ The final dataset contained 428,619 CpG sites.

Methylation $\beta$ values were normalized according to Horvath's modified beta-mixture quantile (BMIQ) normalization.

DNAm GA was calculated as described elsewhere, ${ }^{10}$ based on the methylation profile of 148 selected CpG sites. Chronological GA was based on ultrasound scans, and epigenetic GA was calculated as the arithmetic difference between DNAm GA and chronological GA and adjusted for chronological GA. Adjustment for chronological GA was necessary to remove the effect of chronological GA entirely (Pearson correlation between DNAm GA-GA arithmetic difference and GA $r=-0.27, p<0.01)$.

\section{Child Cord Blood Cell Counts at Birth}

To control for the potential effects of cell type heterogeneity in fetal umbilical cord blood, cord blood cell counts at birth were estimated for 7 cell types (nucleated red blood cells, granulocytes, monocytes, natural killer cells, B cells, CD4 $+\mathrm{T}$ cells, and CD8 $+\mathrm{T}$ cells) using the method of Bakulski et al., ${ }^{18}$ which is also incorporated in the R-package minfi. ${ }^{16}$

\section{Child Genotyping and Multidimensional Scaling Analysis}

To control for the potential effects of population structure, genotyping was performed on Illumina Human Omni Express Exome Arrays (Illumina Inc., San Diego, CA). Only markers with a call rate of at least 98\%, minor allele frequency of $1 \%$ and a $p$ value for deviation from Hardy-Weinberg equilibrium $>1 \times 10^{-06}$ were kept in the analysis. We performed multidimensional scaling (MDS) analysis on the identity by state matrix of quality-controlled genotypes. ${ }^{19}$ The first 2 MDS components depicted the population structure.

\section{Maternal History of Depression Diagnosed Before Pregnancy and Antenatal Depressive Symptoms}

Between 12+0-13+6 weeks + days of gestation, the women answered the question "Have you ever been diagnosed by a physician with depression?" followed by a question on timing of the diagnosis. A total of 76 women reported that the diagnosis occurred before pregnancy ( 3 women who did not specify timing were excluded). Of them, 12 indicated using antidepressant medication during the past year. A total of 618 women reported no history of physician-diagnosed depression and no antidepressant medication use in the past year (3 women who reported using antidepressants but no depression diagnoses were excluded).

Starting from $12+0-13+6$ weeks + days of gestation, each woman completed the 20 item Center for Epidemiological Studies Depression Scale $(\text { CES-D })^{20}$ for depressive symptoms over the previous 7 days biweekly up to 14 times until $38+0-39+6$ weeks + days of gestation or delivery. On average, each woman filled in the CES-D $12.6(\mathrm{SD}=2.2)$ times; of the 581 women with CES-D data, 481 (82.8\%) had less than 2 missing values. We used raw data to calculate trimester-specific means of 
TABLE 1 Sample Characteristics

\begin{tabular}{|c|c|c|c|c|c|c|c|c|c|c|}
\hline \multicolumn{2}{|c|}{$\begin{array}{l}\text { Entire Sample With Fetal } \\
\text { Cord Blood DNA } \\
\text { Methylation Data (N = 814) }\end{array}$} & \multicolumn{3}{|c|}{$\begin{array}{l}\text { Sample With Fetal Cord Blood DNA } \\
\text { Methylation and Maternal History } \\
\text { of Depression Diagnosed Before } \\
\text { Pregnancy Data ( } n=694)\end{array}$} & \multicolumn{3}{|c|}{$\begin{array}{l}\text { Sample With Fetal Cord } \\
\text { Blood DNA Methylation and } \\
\text { Maternal Antenatal Depressive } \\
\text { Symptoms Data ( } \mathbf{n}=\mathbf{5 8 1})\end{array}$} & \multicolumn{3}{|c|}{$\begin{array}{l}\text { Sample With Fetal Cord Blood DNA } \\
\text { Methylation and Child's Psychiatric } \\
\text { Problems in Early Childhood } \\
\text { Follow-up Data ( } n=407)\end{array}$} \\
\hline Mean (SD) or $n(\%)$ & Range & Mean (SD) or $n(\%)$ & Range & p1 & Mean (SD) or $n(\%)$ & Range & p2 & Mean (SD) or $n(\%)$ & Range & p3 \\
\hline $39.8(1.6)$ & $31.0-42.7$ & $39.8(1.6)$ & $31.0-42.7$ & .23 & $39.7(1.6)$ & $31.0-42.7$ & .48 & $39.8(1.5)$ & $31.0-42.7$ & .36 \\
\hline $38.5(2.0)$ & $28.5-47.1$ & $38.5(2.0)$ & $29.6-47.1$ & .28 & $38.4(2.1)$ & $28.5-47.1$ & .18 & $38.6(2.0)$ & $29.6-47.1$ & .035 \\
\hline$-1.3(1.8)$ & $-10.6-7.0$ & $-1.3(1.8)$ & -10.1 to 7.0 & .86 & $-1.4(1.9)$ & -10.6 to 7.0 & .37 & $-1.2(1.9)$ & -8.0 to 7.0 & .12 \\
\hline 430 (52.8\%) & & 365 (52.6\%) & & .41 & 299 (51.5\%) & & .12 & $212(47.9 \%)$ & & .36 \\
\hline $47(5.9 \%)$ & & $40(5.8 \%)$ & & .53 & $34(5.9 \%)$ & & .44 & $22(5.4)$ & & .35 \\
\hline $76(11.0 \%)$ & & $76(11.0 \%)$ & & 1.00 & $62(11.3 \%)$ & & .29 & 49 (12.9\%) & & .11 \\
\hline $11.5(6.5)$ & $0.7-39.9$ & $11.5(6.6)$ & $0.7-39.9$ & .61 & $11.5(6.5)$ & $0.7-39.9$ & 1.00 & $11.5(6.7)$ & $0.7-39.9$ & .86 \\
\hline 139 (17.1\%) & & 119 (17.1\%) & & .39 & $96(16.5 \%)$ & & .35 & 76 (18.7\%) & & .13 \\
\hline $8(1.0 \%)$ & & $6(0.9 \%)$ & & .13 & $6(1.0 \%)$ & & .49 & $3(0.7 \%)$ & & .24 \\
\hline $11(1.4 \%)$ & & 8 (1.2\%) & & .20 & $9(1.5 \%)$ & & .36 & $7(1.7 \%)$ & & .28 \\
\hline
\end{tabular}

Gestational age (wk)

DNAm gestational age (wk)

Epigenetic gestational age

(DNAm gestational age -

gestational age) (wk)

Sex (boys)

1-min Apgar score $\leq 6$

Maternal Characteristics

History of depression

diagnosed before

pregnancy (yes)

Antenatal trimester-weighted

mean CES-D score (0-60)

Age over 40 years at

delivery (yes)

Insulin-treated gestational

diabetes mellitus in previous

pregnancy (yes)

Sjögren syndrome (yes)

Note: $\mathrm{p} 1$ Refers to $\mathrm{p}$ value comparing the sample with data on history of depression diagnosed before pregnancy $(n=694)$, $\mathrm{p} 2$ on maternal antenatal depressive symptoms $(n=581)$, and $\mathrm{p} 3$ on child's psychiatric problems $(n=407)$ with the entire sample $(N=814)$. CES-D = Center for Epidemiological Studies Depression Scale; DNAm = DNA methylation. 
the CES-D sum-scores to represent the level of depressive symptoms at each pregnancy trimester (one value at gestational weeks+days $12+0-13+6$; mean of values between $14+0-27+6$ gestational weeks+days; mean of values between $28+0-39+6$ gestational weeks + days). We then calculated the mean of these 3 trimester-specific values, the trimester-weighted mean value, to represent the overall level of antenatal depressive symptoms.

\section{Child Psychiatric Problems}

When their children were 2.3 to 5.8 years old (mean 3.7, SD 0.75), mothers completed the Child Behavior Checklist (CBCL $1 \frac{1}{2}-5$ ) on psychiatric problems. ${ }^{21} \mathrm{We}$ calculated the total problems $t$ score based on the 99 problem items rated on a scale from 0 (not true) to 2 (very or often true). ${ }^{21} \mathrm{We}$ also calculated internalizing and externalizing problems $t$ scores, 2 subscales of the total problems. ${ }^{21}$

\section{Covariates}

All analyses were adjusted for child's chronological GA, cord blood cell type composition and the first two MDS components. Based on our previous findings in this cohort, ${ }^{11}$ we thereafter made adjustments for maternal age at delivery ( $\geq 40$ years $/<40$ years), insulin-treated gestational diabetes mellitus in a previous pregnancy (yes/no), Sjögren syndrome (yes/no), neonate's 1-minute Apgar score $(\leq 6 />6)$, and sex (girl/ boy). In the analyses of child psychiatric problems, we made further adjustments for child's age at follow-up (years) and maternal Beck Depression Inventory-II scores (BDI-II $<14$ /BDI-II $\geq 14)^{22}$ reported at the child follow-up.

\section{Statistical Analyses}

We studied the associations of maternal history of depression diagnosed before pregnancy and trimester-weighted mean of antenatal depressive symptoms with child epigenetic GA using linear regression analysis. We tested whether these effects were additive by adding maternal depression diagnosis $\times$ trimester-weighted mean of antenatal depressive symptoms interaction term into regression equation following main effects of these variables. We examined gestation stage-specific effects with generalized additive mixed models using a reverse temporal model $^{23}$ that treated the biweekly measures of antenatal depressive symptoms as the longitudinal dependent variable and the child's epigenetic GA as the time-invariant and gestation week as the time-varying independent continuous variables; time (gestation week) $\times$ epigenetic GA interaction tested whether the effect was gestation-stage-specific. For all these analyses, depressive symptoms scores were square root-transformed to attain normality; the symptom scores were further standardized to a mean of 0 and an SD of 1 to facilitate interpretation (for mixed models, the SD scores were calculated using the grand mean of the biweekly CES-D sum-scores across 12 to 39 gestational weeks and its SD to retain within-time variation).

As child's CBCL psychiatric problems scores are not independent of each other but, rather, represent a hierarchical structure, we first examined associations of child's epigenetic GA with early childhood total problems and then examined associations with child's internalizing and externalizing problems using linear regression analyses. Problem scores were logarithm transformed to obtain normality and standardized to facilitate interpretation. In our study, the 2 CBCL subscales were highly intercorrelated (Pearson $r=0.62, p<.001$ ), and together explained $95.3 \%$ of the variance in total problems. However, to decrease the likelihood of type 1 error, we tested the associations with the 2 subscales only if the higher level association was significant and report also Bonferroni-corrected CIs.

In testing whether the associations varied by sex, we entered sex $x$ history of depression diagnosed before pregnancy/antenatal depressive symptoms interaction term into the regression equation with child epigenetic GA at birth as the outcome, and we entered sex $\times$ child epigenetic GA at birth interaction into the regression equation with child psychiatric problems as the outcome.

Finally, we tested whether child epigenetic GA at birth mediated the association between maternal antenatal depression and child psychiatric problems by using the PROCESS macro for SPSS (version 24.0). ${ }^{24}$ Mediation tests were conducted pending these variables were interrelated.

To validate our findings, we applied the bootstrapping method in all analyses. We used unrestricted random sampling method to generate 1,000 samples, and $95 \%$ bootstrap CI (95\% CI) using the normal distribution theory; to account for type 1 error in analyses on internalizing and externalizing problems, we also used Bonferroni-corrected $95 \% \mathrm{CI}(97.5 \% \mathrm{CI}$ is significant at $95 \% \mathrm{CI}$ after correction for 2 tests on subscales). Statistical analyses were performed using IBM SPSS 24.0 and SAS 9.4 .

\section{RESULTS}

Table 1 shows that the subsamples with data on maternal history of depression diagnosed before pregnancy $(\mathrm{n}=694)$, maternal antenatal depressive symptoms $(\mathrm{n}=581)$, and child psychiatric problems $(n=407)$ did not differ from the entire sample in any of the studied characteristics, except that DNAm GA was higher in the subsample with child psychiatric problems data. Pearson correlations between DNAm GA and GA were $0.51(p<.001)$ in the entire sample, and 0.52 $(p<.001), 0.50(p<.001)$, and $0.47(p<.001)$ in the 3 subsamples of participants with data on maternal history of depression diagnosed before pregnancy, data on maternal antenatal depressive symptoms, and data on child psychiatric problems, respectively.

\section{Maternal Antenatal Depression and Child's Epigenetic GA at Birth}

Figure 1 shows that maternal history of depression diagnosed before pregnancy (Figure 1A; exclusion of antidepressant users $(n=12)$ did not alter the finding, $95 \% \mathrm{CI}-0.47$ to -0.01 ) and greater antenatal depressive symptoms (Figure 1B) were associated with lower epigenetic GA after adjusting for child's chronological GA, fetal cord blood cell types, and 2 MDS components (model 1). When adjusted further for the other covariates (model 2), this latter association became nonsignificant (Figure 1).

Furthermore, we found that although child's epigenetic GA was lower for children whose mothers had a depression diagnosis, clinically relevant antenatal depressive symptoms, or both than for children whose mothers reported neither, the effects of maternal history of depression diagnosed before pregnancy and antenatal depressive symptoms were not additive (see Figure S2, available online; women with a depression diagnosis before pregnancy had 3.61 times higher odds $[95 \% \mathrm{CI}=$ 2.09-6.27] of clinically relevant antenatal depressive symptoms [trimester-weighted mean depressive symptoms score $\geq 16$ ] than those without the diagnosis).

There were no gestation stage-specific effects of antenatal depressive symptoms on child's epigenetic GA (see Figure S3, available online).

Finally, we tested whether child's sex moderated any of the associations. There were no significant sex $\times$ maternal depression diagnosis $(95 \% \mathrm{CI}=-0.41$ to 0.46 for interaction term) or sex $\times$ maternal depressive symptoms $(95 \% \mathrm{CI}=-0.07$ to 0.22 for interaction term) interactions on child's epigenetic GA. 
FIGURE 1 Maternal Antenatal Depression and Child's Epigenetic Gestational Age at Birth

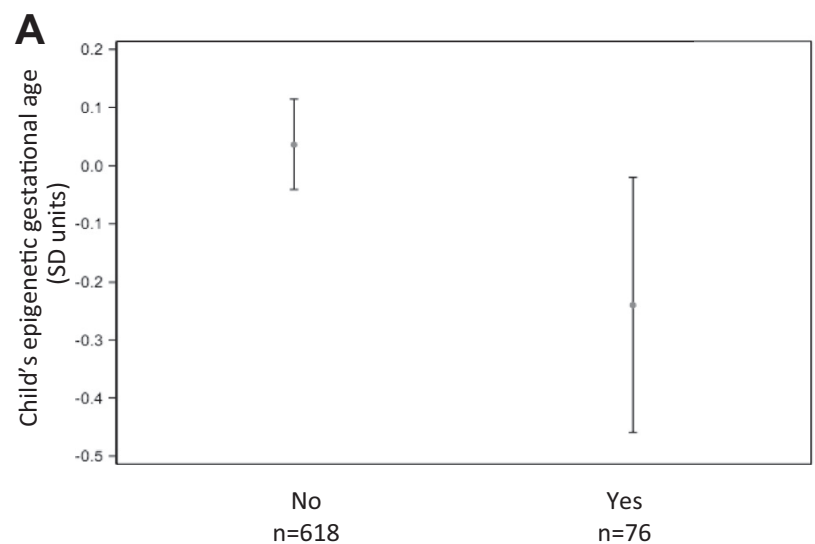

Maternal history of depression diagnosed before pregnancy

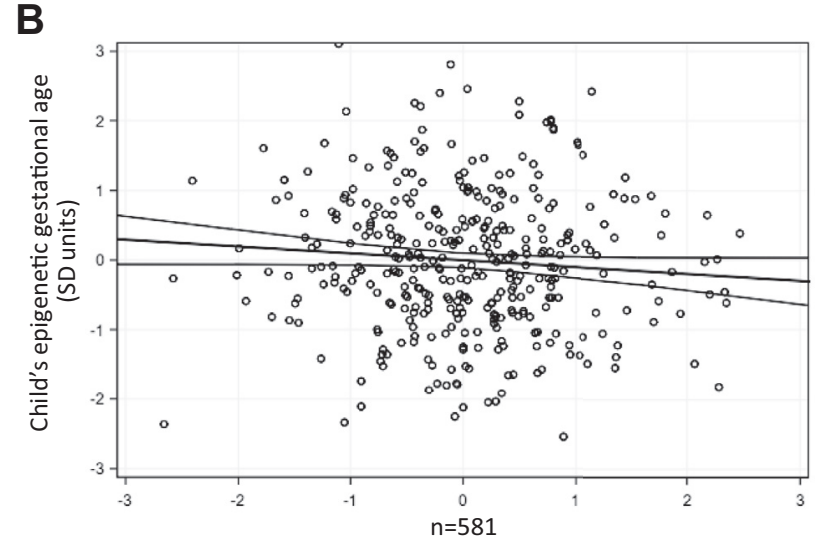

Maternal antenatal trimester-weighted mean depressive symptoms score (SD units)

Note: (A) Means and $95 \% \mathrm{Cls}$ of child's epigenetic gestational age in groups divided according to maternal history of depression diagnosed before pregnancy. The unstandardized regression coefficients $(\beta)$ and $95 \% \mathrm{Cls}$ refer to mean differences in epigenetic gestational age between children of mothers with and without a history of depression diagnosed before pregnancy derived from linear regression analyses. $\beta=-0.25,95 \% \mathrm{Cl}=-0.46$ to -0.03 (model 1 ); $\beta=-0.24,95 \%$ $\mathrm{Cl}=-0.45$ to -0.02 (model 2).

(B) Scatterplot with a regression line displaying the unstandardized regression coefficient $(\beta)$ and $95 \% \quad C l s$ showing associations between maternal trimester-weighted mean antenatal depressive symptoms and child's epigenetic gestational age. $\beta=-0.08,95 \% \mathrm{Cl}=-0.16$ to -0.004 (model 1 ); $\beta=-0.07,95 \%$ $\mathrm{Cl}=-0.15$ to 0.004 (model 2).

( $A$ and $B$ ) Regression model 1 is adjusted for child's chronological gestational age at birth, fetal cord blood cell type composition, and 2 multidimensional scaling components; model 2 for model 1 plus maternal age at delivery, insulin-treated gestational diabetes mellitus in previous pregnancy, Sjögren syndrome, child's 1-minute Apgar score, and sex. Figures display Figures $1 \mathrm{~A}$ and $1 \mathrm{~B}$.

\section{Child's Epigenetic GA at Birth and Psychiatric Problems at 3.7 Years}

Table 2 shows that there were no significant associations between child's epigenetic GA and psychiatric problems in the total sample. However, sex $\times$ epigenetic GA interactions were significant for total and internalizing problems (Table 2). In model 2, there was an interaction also on externalizing problems. The interaction between sex and epigenetic GA on internalizing problems remained significant after Bonferroni correction for 2 conducted tests (Bonferroni-corrected 95\% CI $=0.02-0.48$ and $0.01-0.46$ in models 1 and 2, respectively), whereas that on externalizing problems in model 2 did not (Bonferroni-corrected 95\% $\mathrm{CI}=-0.02$ to 0.43 ).

Table 2 shows that, in boys but not in girls, lower epigenetic GA at birth was significantly associated with greater total and internalizing problems. In neither boys nor in girls was epigenetic GA at birth significantly associated with externalizing problems (Table 2). Furthermore, Table S1 (available online) shows sample characteristics by sex and highlights that, on average, boys had significantly lower epigenetic GA at birth and higher total and externalizing problems scores than girls.

\section{Associations Between Maternal Antenatal Depression and Child's Psychiatric Problems at 3.7 Years: Mediation via Child's Epigenetic GA}

The mediation analyses were conducted in boys, as epigenetic GA was not associated with psychiatric problems in girls; and only on total and internalizing problems, as they were predicted by child's epigenetic GA (Table 2) and by maternal antenatal depression in boys (see Table S2, available online). Figure 2 shows that, in boys, epigenetic GA partially mediated the association between antenatal depression and internalizing problems. Mediation was not significant on total problems (95\% $\mathrm{CI}=-0.001$ to 0.14 for indirect effect).

\section{DISCUSSION}

This study shows that maternal history of depression diagnosed before pregnancy and higher levels of antenatal depressive symptoms were significantly associated with child's lower epigenetic GA at birth. The effects of these conditions were not additive, and antenatal symptoms effects were not gestation stage specific. This study also shows that child's lower epigenetic GA at birth prospectively predicted child's total and internalizing problems in early childhood, although only in boys. Also in boys, child's epigenetic GA at birth partially mediated the association of maternal antenatal depression with child internalizing problems.

These findings may seem to contradict the findings in adult populations that have shown that higher rather than lower epigenetic age, in comparison to chronological age, is associated with several negative health outcomes. $^{8,25,26}$ That lower epigenetic age might indicate such an increased risk is, however, congruent with the $\mathrm{DOHaD}$ concept: the risk for aging-related diseases and mental problems is increased in individuals exposed to prenatal environmental adversities, which are associated with lower birth weight, lower GA, and maternal depression during pregnancy. $^{1,2}$ Because DNA methylation has been shown to undergo age-related changes, ${ }^{27}$ it remains possible that both lower and higher epigenetic GA signal risk. This risk may, however, depend on life stage. Future studies will need to evaluate this hypothesis and whether epigenetic GA at birth predicts other epigenetic age biomarkers in later life.

Although we expected to find sex differences in the associations, it remains elusive why the associations with psychiatric problems were specific to boys. It has been suggested that sex differences in the "programming effects" of prenatal stress may serve an adaptive, evolutionary purpose. In stressful environments, it may be more adaptive for females to be vigilant, alert, and stress responsive to protect the young; this may become phenotypically apparent in depression and anxiety. ${ }^{5}$ For males, aggression and stress resistance may be more adaptive as they go out, explore new environments, and compete with other males. ${ }^{5}$ These "adaptive" phenotypes may become vulnerabilities when they no longer 
TABLE 2 Associations Between Child's Epigenetic Gestational Age at Birth and Psychiatric Problems at 3.7 Years

Epigenetic Gestational Age in SD Units (Independent Predictor Variable)

Psychiatric Problems in SD Units: (Dependent Outcome Variable)

Total

Model 1

Model 2

Internalizing

Model 1

Model 2

Externalizing

Model 1

Model 2

\begin{tabular}{|c|c|c|}
\hline \multicolumn{2}{|c|}{$\begin{array}{l}\text { Epigenetic } \\
\text { Gestational Age } \\
\text { Main Effect }^{\mathrm{a}}\end{array}$} & \multirow{2}{*}{$\begin{array}{c}\text { Sex } \times \begin{array}{c}\text { Epigenetic Gestationa } \\
\text { Age Interaction }\end{array} \\
95 \% \mathrm{Cl}^{\mathrm{b}}\end{array}$} \\
\hline$\beta^{a}$ & $95 \% \mathrm{Cl}^{\mathrm{a}}$ & \\
\hline-0.06 & -0.17 to 0.05 & 0.04 to 0.44 \\
\hline-0.06 & -0.16 to 0.05 & 0.06 to 0.44 \\
\hline-0.04 & -0.15 to 0.08 & 0.05 to 0.46 \\
\hline-0.03 & -0.15 to 0.08 & 0.03 to 0.43 \\
\hline-0.06 & -0.17 to 0.05 & -0.01 to 0.39 \\
\hline-0.06 & -0.17 to 0.05 & 0.01 to 0.40 \\
\hline
\end{tabular}

\begin{tabular}{|c|c|c|c|}
\hline \multicolumn{2}{|c|}{ Boys $(n=212)$} & \multicolumn{2}{|c|}{ Girls $(n=195)$} \\
\hline$\beta$ & $95 \% \mathrm{Cl}$ & $\beta$ & $95 \% \mathrm{Cl}$ \\
\hline-0.16 & -0.31 to -0.0003 & 0.08 & -0.08 to 0.24 \\
\hline-0.17 & -0.32 to -0.02 & 0.10 & -0.06 to 0.26 \\
\hline-0.16 & -0.31 to 0.001 & 0.13 & -0.05 to 0.30 \\
\hline-0.16 & -0.31 to -0.01 & 0.14 & -0.04 to 0.31 \\
\hline-0.13 & -0.29 to 0.02 & 0.06 & -0.10 to 0.23 \\
\hline-0.15 & -0.30 to 0.01 & 0.08 & -0.08 to 0.24 \\
\hline
\end{tabular}

Note: $\beta$ refers to unstandardized regression coefficient from linear regression analysis, indicating SD change in problem scores per SD change in epigenetic gestational age. Model 1 is adjusted for child's chronological gestational age, fetal cord blood cell counts, 2 multidimensional scaling components from genome-wide data, child's sex (pooled analysis), and age at follow-up. Model 2 is adjusted for model 1 covariates plus maternal age at delivery, insulin-treated gestational diabetes mellitus in previous pregnancy, Sjögren syndrome, maternal Beck Depression Inventory-II score parallel to child rating, and child's 1-minute Apgar score.

${ }^{a}$ Main effects in the total sample are tested without sex $\times$ epigenetic gestational age interaction term, whereas sex $\times$ epigenetic gestational age interactions are tested in the presence of main effects of these predictor variables.

'These Cls pertain to the interaction term "sex $\times$ epigenetic gestational age" tested in the presence of main effects.

match the environments. ${ }^{14}$ The association that we found with the internalizing phenotype in boys contradicts the suggested evolutionarily adaptive male phenotype. It also contradicts previous findings showing that maternal depression during pregnancy predicts depression specifically in girls. ${ }^{4}$ However, many studies suggest corresponding adverse effects of antenatal depression on offspring psychopathology in both sexes, ${ }^{3,5}$ and, corresponding with our findings, a rodent study showed that maternal early pregnancy stress predicted a depression-like phenotype only in male offspring. ${ }^{14}$ Indeed, evidence exists suggesting that what might be phenotypically adaptive for males and females may be

FIGURE 2 Maternal Antenatal Depression Acts Partly via Child's Epigenetic Gestational Age at Birth on Child's Psychiatric Problems Among Boys

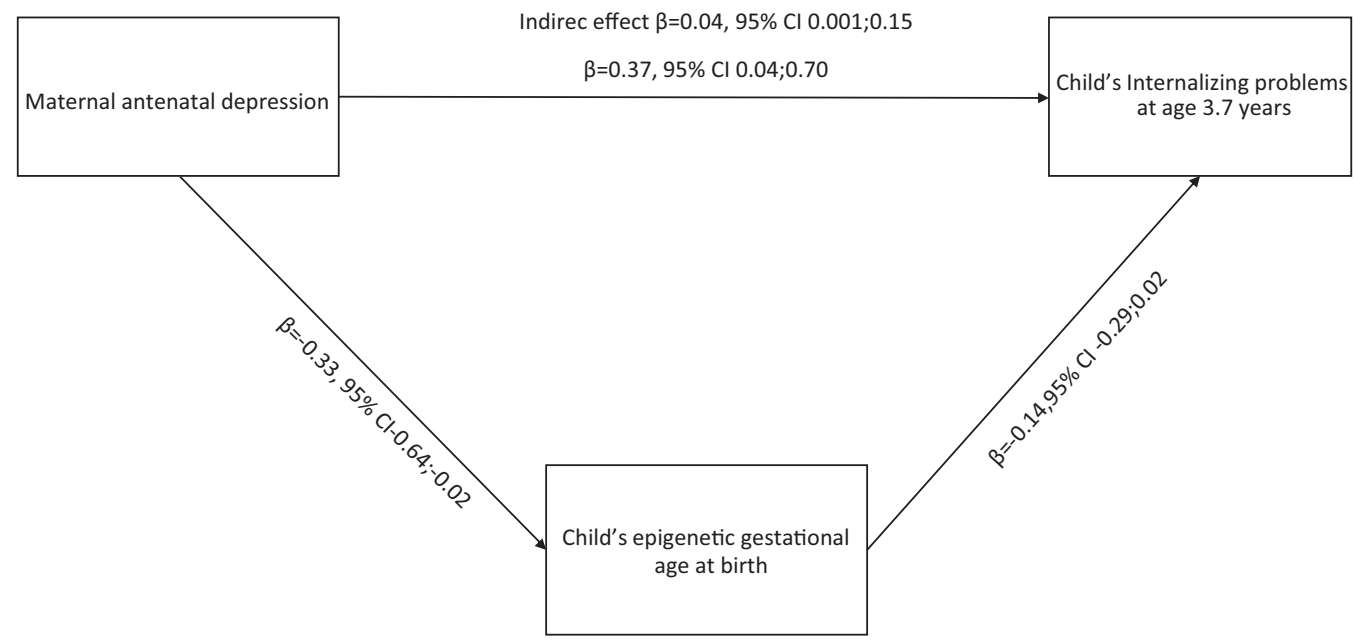

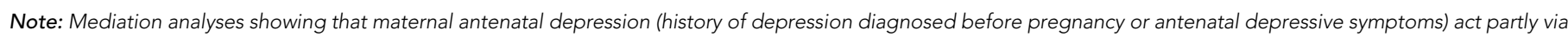

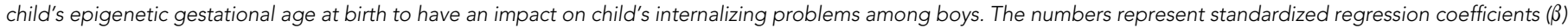

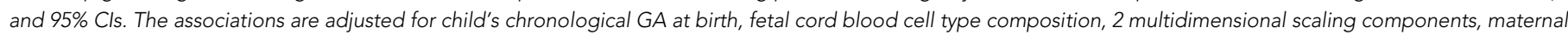

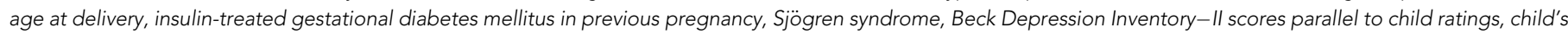
1-minute Apgar score, and age at early childhood testing. 
determined by a complex interaction of type, timing, severity, and chronicity of the prenatal stress exposure and the developmental stage of the offspring. ${ }^{5,14}$ Whether lower epigenetic GA in boys would also predict other outcomes or would become predictive of outcomes in girls in later life stages is the subject of ongoing studies. Furthermore, since we found that boys had lower epigenetic GA at birth and higher total and externalizing psychiatric problems in early childhood than girls, the sex differences in the associations of epigenetic GA and psychiatric problems may also possibly have resulted from a greater ability to detect such associations in boys. Yet, we found no sex differences in the levels of internalizing problems, but epigenetic GA and internalizing problems were also associated only in boys. The findings on the possible sex specificity of the effects of prenatal stress on offspring developmental outcomes are inconclusive, and a consensus exists that further exploration of the potential sex specificity of prenatal programming effects is needed. ${ }^{5,14}$

The main study strength pertains to the well-characterized, ethnically homogenous cohort and prospective mother-child data. Chronological GA was based on ultrasound scans, and we applied novel bioinformatics methods to account for any sample contamination by maternal blood ${ }^{17}$ and for the effects of cell type heterogeneity ${ }^{18}$ and genetic population structure, ${ }^{19}$ which are factors strongly influencing epigenetic profiles. ${ }^{28}$ Furthermore, we validated our findings by using the bootstrap method and applied a hierarchically structured analysis strategy when we examined child psychiatric problems to decrease the likelihood of false-positive findings. We also corrected these analyses for multiple testing, and the sex interaction on internalizing problems remained significant. Yet, our novel findings must be interpreted with caution, and further studies are needed to confirm or refute them.

The limitations of our study relate to our recruitment strategy, which was based on women's risk factor status for preeclampsia and IUGR, the ethnic homogeneity of our sample, and the use of one tissue type, precluding generalizations to groups that differ from ours and other tissues. Sample attrition limits the generalizability of our study findings, and the low number of mothers with depression diagnosis limited our statistical power to assess additive effects of depression before and during pregnancy. Also, the biological mechanisms driving the effects found remain unclear. During pregnancy, these mechanisms may include depression-related endocrine factors, neurotransmitters, and levels of inflammatory markers in the mother, fetus, and placenta. ${ }^{29}$ In fact, epigenetic age acceleration in adults may be driven by direct effects of glucocorticoids on DNA methylation. ${ }^{30}$ The mechanisms involved after birth may include structural and functional immaturity of organs and problems in neonatal adaptation increasing vulnerability to

\section{REFERENCES}

1. Lahti M, Savolainen K, Tuovinen S, et al. Maternal depressive symptoms during and after pregnancy and psychiatric problems in children. J Am Acad Child Adolesc Psychiatry. 2017;56:30-39.

2. Pesonen A-K, Lahti M, Kuusinen T, et al. Maternal prenatal positive affect, depressive and anxiety symptoms and birth outcomes: the PREDO study. PLoS One. 2016;11:2.

3. Van den Bergh BRH, van den Heuvel MI, Lahti M, et al. Prenatal developmental origins of behavior and mental health: the influence of maternal stress in pregnancy. Neurosci Biobehav Rev. 2017 Jul 28. pii: S0149-7634(16)30734-5; https://doi.org/10.1016/j. neubiorev.2017.07.003 [Epub ahead of print].

4. Quarini C, Pearson RM, Stein A, Ramchandani PG, Lewis G, Evans J. Are female children more vulnerable to the long-term effects of maternal depression during pregnancy? J Affect Disord. 2016;189:329-335.

5. Glover V, Hill J. Sex differences in the programming effects of prenatal stress on psychopathology and stress responses: an evolutionary perspective. Physiol Behav. 2012;106: 736-740. developmental adversities. Maternal depression after pregnancy may also compromise the developing attachment bond. However, in our study, maternal depression parallel to rating the child did not account for the associations found between child epigenetic GA at birth and child psychiatric problems.

In summary, children of mothers with a history of depression diagnosed before pregnancy or with greater antenatal depressive symptoms display lower epigenetic GA at birth. This lower epigenetic GA at birth seems to be associated with a developmental disadvantage for boys, who went on to display greater psychiatric problems in early childhood. Lower epigenetic GA actually partially mediated the effects of maternal depression on child outcome. Epigenetic GA at birth may hold potential for identifying, before the onset of psychiatric problems, at-risk children, thereby allowing for timely, personalized preventive interventions.

\section{Accepted March 9, 2018.}

Drs. Lahti, Lahti-Pulkkinen, Räikkönen, Mss. Suarez and Girchenko, and Mr. Lipsanen are with the University of Helsinki, Finland. Drs. Czamara and Binder are with the Max-Planck Institute of Psychiatry, Germany. Drs. Hämäläinen, Laivuori, and Villa are with the University of Helsinki and Helsinki University Hospital, Finland. Dr. Kajantie is with the National Institute for Health and Welfare, Helsinki, Finland. Dr. Reynolds is with the Queen's Medical Research Institute, University of Edinburgh, UK. Dr. Smith and Ms. Knight are with Emory University, Atlanta, GA.

This work was supported by the Academy of Finland, EraNet Neuron, EVO (a state subsidy for health science research), Doctoral Programme in Psychology, Learning and Communication, and the University of Helsinki.

A preliminary study abstract was presented at the International Society of Psychoneuroendocrinology's $46^{\text {th }}$ Annual Conference in Miami, FL, September $8-11,2016$, and at the International Society of Psychiatric Genetics' $25^{\text {th }}$ World Congress in Orlando, FL, October 13-17, 2017, and was published in Psychoneuroendocrinology 2016;71S:63.

The authors thank Jessica Keverne, PhD, Max-Planck Institute of Psychiatry, Germany, for manuscript editing.

Disclosure: Drs. Lahti, Czamara, Lahti-Pulkkinen, Hämäläinen, Kajantie, Laivuori, Villa, Reynolds, Smith, Binder, Räikkönen, and Mss. Suarez, Knight, Girchenko, and Mr. Lipsanen report no biomedical financial interests or potential conflicts of interest.

Correspondence to Marius Lahti-Pulkkinen, PhD, Department of Psychology and Logopedics, University of Helsinki, Haartmaninkatu 3, PO Box 21, Fl-00014 Finland; e-mail: marius.lahti-pulkkinen@helsinki.fi

0890-8567/\$36.00/๑2018 American Academy of Child and Adolescent Psychiatry

https://doi.org/10.1016/j.jaac.2018.02.011

6. Hannum G, Guinney J, Zhao L, et al. Genome-wide methylation profiles reveal quantitative views of human aging rates. Mol Cell. 2013;49:359-367.

7. Horvath S. DNA methylation age of human tissues and cell types. Genome Biol. 2013; 14:R115.

8. Chen BH, Bressler J, Fornage M, et al. DNA methylation-based measures of biological age: meta-analysis predicting time to death. Aging (Albany NY). 2016;8:1844-1865.

9. Simpkin AJ, Hemani G, Suderman M, et al. Prenatal and early life influences on epigenetic age in children: a study of mother-offspring pairs from two cohort studies. Hum Mol Genet. 2016;25:191-201.

10. Knight AK, Craig JM, Theda C, et al. An epigenetic clock for gestational age at birth based on blood methylation data. Genome Biol. 2016;17:206.

11. Girchenko P, Lahti J, Czamara D, et al. Associations between maternal risk factors of adverse pregnancy and birth outcomes and the offspring epigenetic clock of gestational age at birth. Clin Epigenetics. 2017;9:1-15.

12. Austad SN, Fischer KE. Sex differences in lifespan. Cell Metab. 2016;23:1022-1033. 
13. Glasheen C, Richardson GA, Kim KH, Larkby CA, Swartz HA, Day NL. Exposure to maternal pre- and postnatal depression and anxiety symptoms: risk for major depression, anxiety disorders, and conduct disorder in adolescent offspring. Dev Psychopathol. 2013; 25:1045-1063.

14. Mueller BR, Bale TL. Sex-specific programming of offspring emotionality after stress early in pregnancy. J Neurosci. 2008;28:9055-9065.

15. Girchenko P, Lahti M, Tuovinen S, et al. Cohort profile: Prediction and Prevention of Preeclampsia and Intrauterine Growth Restriction (PREDO) study. Int J Epidemiol. 2017;46:1380-1381

16. Aryee MJ, Jaffe AE, Corrada-Bravo H, et al. Minfi: a flexible and comprehensive Bioconductor package for the analysis of Infinium DNA methylation microarrays. Bioinformatics. 2014;30:1363-1369.

17. Morin AM, Gatev E, McEwen LM, et al. Maternal blood contamination of collected cord blood can be identified using DNA methylation at three CpGs. Clin Epigenetics. 2017; 9:1-9.

18. Bakulski KM, Feinberg JI, Andrews SV, et al. DNA methylation of cord blood cell types: applications for mixed cell birth studies. Epigenetics. 2016;11:354-362.

19. Barfield RT, Almli LM, Kilaru V, et al. Accounting for population stratification in DNA methylation studies. Genet Epidemiol. 2014;38:231-241.

20. Radloff LS. The CES-D Scale: a self-report depression scale for research in the general population. Appl Psychol Meas. 1977;1:385-401.

21. Achenbach TM, Rescorla LA. Manual for the ASEBA Preschool Forms \& Profiles. Burlington, VT; 2000.
22. Beck A, Steer R, Brown G. BDI-II, Beck Depression Inventory: Manual. San Antonio, TX: Psychological Corporation; 1996.

23. Chen YH, Ferguson KK, Meeker JD, McElrath TF, Mukherjee B. Statistical methods for modeling repeated measures of maternal environmental exposure biomarkers during pregnancy in association with preterm birth. Environ Health. 2015;14:9.

24. Hayes AF. Introduction to Mediation, Moderation, and Conditional Process Analysis: A Regression-Based Approach. New York, NY: Guilford Press; 2013.

25. Horvath S, Erhart W, Brosch M, et al. Obesity accelerates epigenetic aging of human liver. Proc Natl Acad Sci U S A. 2014;111:15538-15543.

26. Marioni RE, Shah S, McRae AF, et al. The epigenetic clock is correlated with physical and cognitive fitness in the Lothian Birth Cohort 1936. Int J Epidemiol. 2015;44: 1388-1396.

27. Jung M, Pfeifer GP. Aging and DNA methylation. BMC Biol. 2015;13:7.

28. Lam LL, Emberly E, Fraser HB, et al. Factors underlying variable DNA methylation in a human community cohort. Proc Natl Acad Sci. 2012;109(Suppl 2): 17253-17260.

29. Reynolds RM, Pesonen A-K, O’Reilly JR, et al. Maternal depressive symptoms throughout pregnancy are associated with increased placental glucocorticoid sensitivity. Psychol Med. 2015;45:2023-2030.

30. Zannas AS, Arloth J, Carrillo-Roa T, et al. Lifetime stress accelerates epigenetic aging in an urban, African American cohort: relevance of glucocorticoid signaling. Genome Biol. 2015;16:1-12. 
FIGURE S1 Flow Chart of the Prediction and Prevention of Preeclampsia and Intrauterine Growth Retardation (PREDO) Study and Sample Attrition

876 fetal cord blood DNA samples available for genome-wide methylation analysis using Infinium Human Methylation 450 BeadChip
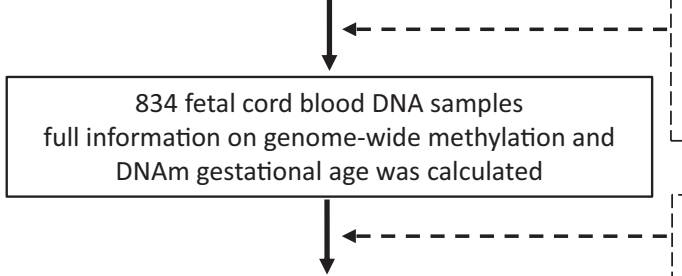

825 fetal cord blood DNA samples passed quality control procedures and had full information on genome-wide methylation

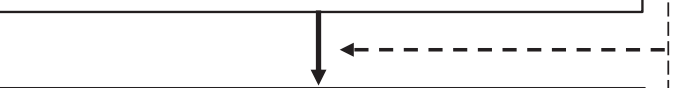

817 fetal cord blood DNA samples valid information on genome-wide methylation and genotype data

\begin{tabular}{|l|}
\hline \\
814 fetal cord blood DNA samples \\
with child epigenetic gestational age
\end{tabular}

with child epigenetic gestational age
42 samples excluded:

duplicates, outliers in the median intensities, crosshybridizing probes or probes containing SNPs and $\mathrm{CpG}$ sites with a detection $P$-value $>0.01$ in at least $50 \%$ of the samples, due to location on chromosome $\mathrm{X}$ or $\mathrm{Y}$, orsex discrepancies

L_- - - - - $\mathrm{X}$ or $Y$, orsex discrepancies

9 samples excluded:
maternal blood contamination

8 samples excluded:

call rate below $98 \%$ and discrepancies between phenotypic and genotypic sex in genome-wide genotype data

3 samples excluded:

missing chronological gestational age data and an outlier with epigenetic gestational age $>5 \mathrm{SD}$

694 with data on maternal history of depression diagnosed before pregnancy

$\mathbf{5 8 1}$ with data on maternal antenatal depressive symptoms

407 with data on child psychiatric problems in early childhood follow-up

TABLE S1 Characteristics of the Sample With Data on Child Psychiatric Problems in Early Childhood Follow-up Stratified by Child's Sex $(n=407)$

\section{Characteristics}

Child Characteristics at Birth and in Early Childhood

Gestational age (wk)

DNAm gestational age (wk)

Epigenetic gestational age (DNAm gestational age gestational age) (wk)

Age at follow-up (y)

1 -min Apgar score $\leq 6$ (yes)

Psychiatric problems

Total

Internalizing

Externalizing

Maternal Characteristics

History of depression diagnosed before pregnancy (yes)

Antenatal trimester-weighted mean score of

$$
\text { CES-D (0-60) }
$$

Age $>40$ y at delivery (yes)

Insulin-treated gestational diabetes mellitus in previous pregnancy (yes)

Sjögren syndrome (yes)

BDI-Il score in early childhood follow-up
Boys $(n=212)$

Mean (SD) or $\mathrm{n}(\%)$

$39.8(1.5)$
$38.3(2.0)$
$-1.5(2.0)$
$3.7(0.8)$
$12(5.7 \%)$
$46.6(9.6)$
$45.3(9.0)$
$48.4(9.5)$
$31(15.5 \%)$
$11.7(6.7)$
$42(19.8 \%)$
$2(0.9 \%)$
$5(2.4 \%)$
$6.8(7.1)$

Girls $(n=195)$

Range

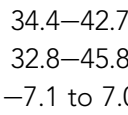

$2.4-5.8$

$28.0-74.0$

$29.0-76.0$

28.0-83.0

$$
44.6 \text { (8.4) }
$$

$44.4(7.9)$

$45.3(7.8)$

$28.0-68.0$

$29.0-70.0$

$31.0-42.0$

$29.6-47.1$

-8.0 to 6.8

$2.3-5.8$

$28.0-67.0$

p Boys vs. Girls

$18(9.9 \%)$

$1.0-39.8$

11.2 (6.7)

$0.7-35.3$

34 (17.4\%)

$1(0.5 \%)$

$2(1.0 \%)$

$0.0-41.0$
.74

.63

.49

.010

.43

$<.001$

071

.49

.31

.53

.26

.39

Note: BDI-II = Beck Depression Inventory-II; CES-D = Center for Epidemiological Studies Depression Scale; DNAm = DNA methylation. 
FIGURE S2 Lack of Additive Effects of Maternal History of

Depression Diagnosed Before Pregnancy and Antenatal

Trimester-Weighted Mean Center for Epidemiological

Studies Depression Scale (CES-D) Score on Child's

Epigenetic Gestational Age (GA) at Birth

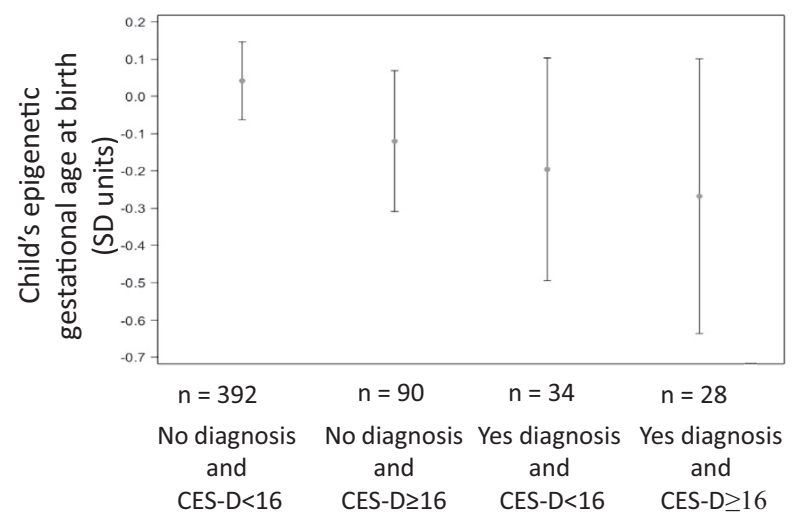

Note: $95 \%=-0.31$ to 0.61 (model 1), $95 \% \mathrm{Cl}=-0.32$ to 0.60 (model 2) for maternal history of depression diagnosed before pregnancy (yes versus no) $x$ antenatal trimester-weighted mean Center for Epidemiological Studies Depression Scale (CES-D) score (CES-D $\geq 16$ versus <16) interaction; mean difference $=-0.18,95 \% \mathrm{Cl}=-0.34$ to $-0.03($ model 1$), 95 \% \mathrm{Cl}=-0.33$ to -0.02 (model 2) for no diagnosis and CES-D $<16$ versus other groups. A total of 37 women who had antenatal depressive symptoms data did not have data on history of depression diagnosed before pregnancy. Model 1 is adjusted for child's chronological GA at birth, fetal cord blood cell type composition, and 2 multidimensional scaling components; and Model 2 for Model 1 covariates plus maternal age at delivery, insulin-treated gestational diabetes mellitus in previous pregnancy, Sjögren syndrome, child's 1-minute Apgar score, and sex.
FIGURE S3 Associations Between Maternal Antenatal

Depressive Symptoms at Different Stages of Pregnancy and

Child's Epigenetic Gestational Age

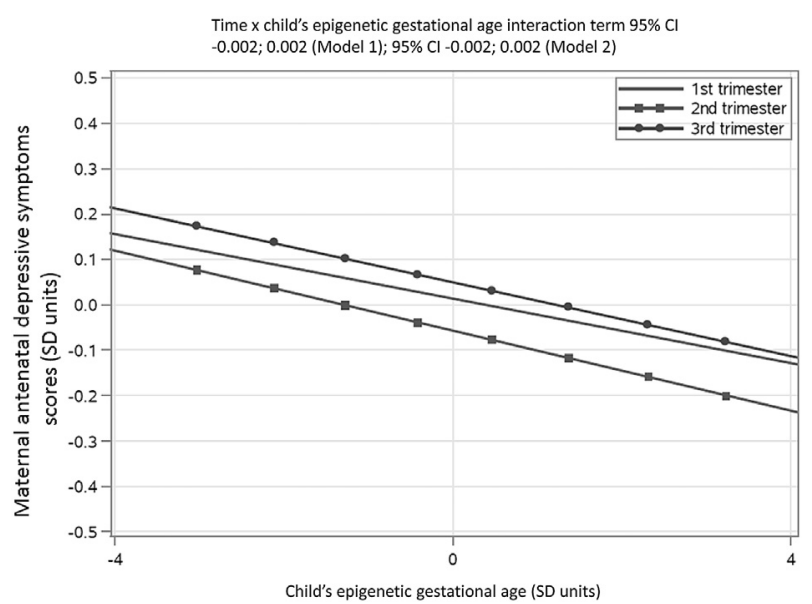

Note: To test whether the associations were gestation stage specific, we used generalized additive mixed models (reverse temporal model) that treated the biweekly measures of antenatal depressive symptoms as the longitudinal dependent variable and epigenetic gestational age as the time-invariant continuous variable and gestation week as the time-varying independent continuous variable; time (gestation week) $\times$ epigenetic gestational age interaction tested whether the effect on biweekly antenatal depressive symptoms was gestation stage specific. For display purposes, regression lines are presented pooling the individual slopes for the first trimester (slopes at 12+0-13+6 weeks of gestation), second trimester (mean of slopes between 14+0-27+6 gestational weeks), and third trimester (mean of slopes between 28+0-39+6 gestational weeks)

TABLE 52 Associations Between Maternal Antenatal Depression (History of Depression Diagnosed Before Pregnancy or Center for Epidemiological Studies Depression Scale Score $\geq 16$ vs. Neither One) and Child's Psychiatric Problems at 3.7 Years

Boys $(n=212)$

Mean Difference: Yes vs.

No Maternal

Child's Psychiatric Problems

Total

Internalizing
$95 \% \mathrm{Cl}$

$0.21-0.81$

$0.16-0.76$
Girls $(n=195)$

\begin{tabular}{cc}
\hline $\begin{array}{c}\text { Mean Difference: Yes vs. } \\
\text { No Maternal }\end{array}$ & \\
Antenatal Depression & $\mathbf{9 5 \%} \mathbf{~ C l}$ \\
0.49 & $0.20-0.78$ \\
0.48 & $0.17-0.80$ \\
\hline
\end{tabular}

Note: Model adjusted for child's age at testing. 